Témoigner Témoigner. Entre histoire et mémoire

Getuigen Revue pluridisciplinaire de la Fondation Auschwitz

$118 \mid 2014$

Au nom des victimes. Dictature et terreur d'État en Argentine, Chili et Uruguay

\title{
Ivan Jablonka et Annette Wieviorka, Nouvelles Perspectives sur la Shoah
}

\section{Anthony Michel}

\section{(2) OpenEdition}

Journals

Édition électronique

URL : http://journals.openedition.org/temoigner/1040

DOI : $10.4000 /$ temoigner. 1040

ISSN : 2506-6390

Éditeur :

Éditions du Centre d'études et de documentation Mémoire d'Auschwitz, Éditions Kimé

Édition imprimée

Date de publication : 1 octobre 2014

Pagination : 186-187

ISBN : 978-2-84174-674-3

ISSN : 2031-4183

Référence électronique

Anthony Michel, «Ivan Jablonka et Annette Wieviorka, Nouvelles Perspectives sur la Shoah », Témoigner. Entre histoire et mémoire [En ligne], 118 | 2014, mis en ligne le 01 octobre 2015, consulté le 23 octobre 2020. URL : http://journals.openedition.org/temoigner/1040 ; DOl : https://doi.org/10.4000/ temoigner. 1040 


\section{$\checkmark$ Librairie}

\section{SECRET D'ENFANCE}

Jean-Claude Snyders
Paris, Le Manuscrit, 2012, 317 p.

enouveau livre de Jean-Claude Snyders prend forme d'un récit. Sur le mode d'une parabole, s'isole dans un paysage, dans des habitudes, dans ses pensées, dans son silence. Bref, il tait sa souffrance. Peut-être lui est-il arrivé des choses si sombres qu' s'abstient de les considérer: «Y Ya-t-il un seul être dont souhaiterait la proximité? », se demande alors l'auteu du livre, frère de centaines de milliers dautres dontles parents ont été déportés dans des camps par les nazis. Curieusement, l'émotion que pourrait éprouver ( étranger, devient pour lui une faiblesse, tout soutien laffection une, pour ne paslappeler une ruse. Même lafection de son enfant lui hinspire de la méflance. Elle risque de le faire changer, de briser son isolement, son refuge. Cet honne, cest, pour ainsi dire, le fantốme de Georges Snyders, le perre de lauteur, déporté

Ce livre pourrait rester un témoignage, celui de ces enfants confrontés au silence de leurs parents sur leur sejour dans les canps de déportation et dextermination nazis. Mais il est bien plus que cela, car il a valeur dintrospection, de plongée en soi-mêne et, a ce lenfance. lenfance. L'enabilité, un premier « péché '́ai-je fait, se deman premier "péché » : quel mal du père : se disque le mal est en moi, même si jence du père: " puisque le mal est en moi, même si je m'y efforce je ne peux pas cesser detre mauvais » [p. 48] Ce fils porte aussi en lui une mission. Celle de vele Ce fils porte aussien lui une mission. Celle de venir à lié, blessé, détruit.

La scène se renverse, plus tard, lorsque l'enfant, devenu père à son tour, est entouré de ses propres nfants et quà leur contact, il repense à cette parti de sa vie. Ce n'est déjà plus sa propre enfance qu’il revisite, mais l'enfance en soi. Ce lieu des espoirs et des désespoirs, des amours et des haines, des désirs et des interdictions, des larmes et des rires. Jean-Claude Snyders développe ainsi, à partir de cette grande souffrance incompréhensible pour lui, une réflexion sur la genèse des passions d'amour et de haine, d'affection et de détestation. Il les tire des plus petites expériences de la vie quotidienne ou des plus grands événement de Phistoire : «Les choses hideuses que j’ai perçue jadis, écrit-il, qui sont entrees en moi, m'ont convaincu de mon horreur profonde; depuis longtemps j'essaie

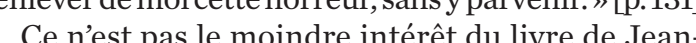
Ce n'est pas le moindre intérét du livre de JeanClaude Snyders que de décrire le chemin qui conduit de ces terribles souvenirs à une forme de dépassement, peut-être de résurection, chemin callouteux, dans des épsage austere, exposé aux vents et aux rafales

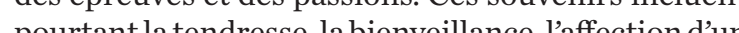
pourtant latendresse, labienveillance,l'affection d'un père pour son enfant. Avec, encreux dans ce message, tout ce qui a manqué à dau

On l'aura compris, le livre de Jean-Claude Snyders se situe au carrefour des Memoires, de la psychologie et de la philosophie de léducation. Mais au plus profon. de lui-même, il est surtout une remontee vers l'espoi pour tous ceux et toutes celles qui ont souffert de la souffrance que les parents leur ont léguee. Le livre se termine sur un texte de Georges Snyders, perre de de la libération du Le Monde pour le cinquantenaire etémoération du canp d'Auschwitz. Texte terrifiant André Rauch

\section{AURAIS-JE ÉTÉ RÉSISTANT \\ OU BOURREAU?}

Pierre Bayard

Paris, Éditions de Minuit, « Paradoxe », 2013, 158 p.

a question qui fait titre en a hanté plus d'un. Dan sa formelle généralité, celle-ci est privée de toute Pierre Bayard la refuse et se l'adresse très directement.
C'est elle et elle seule que son livre examine. Le proogue en guide la lecture.

(1) personnage dont la fonction est de le représenter lui, Pierre Bayard, tel qu'il se transporte, non une généra(ix encén d'entrée à l'́́cole nor co d'Ul dentré à lEcole normale superieure de la rue d Ulm à Paris. Cette transposition et ce déplacement réclament celui que Bayard appelle le personnage-délégué.

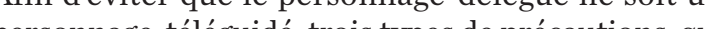
personage-telécuide, trois types de précautions, qu sont autant de protections pour lauteur, sont prises : sermior des lois scientifiques du comportement (behaviorisme, expérience de Milgran et sa critique),

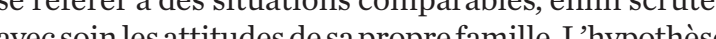
avec soinles attitudesdesaproprén se confhypothè exige que le personnage-delégué se confronte a des des personnes réelles. Elle se conforte rup tres es peron tions vraies auxquelles se plait la fiction que déploie d'un batilon allen d'un batallon allemand qui, en juillet 1942, refusent lordre de commettre le meurtre de masse. Le personnage-délégú reçoitunesortede corporeltépsychique lauteur quion de "personnalté potentielle » que the Piecre à linconscient.

Pierre Bayard inscrit sa démarche et son livre dan un procès defiction. Iltientaunombre de ses mérites pouvoir d approcher une vérite. Comment alors lire ce . D' ó ar Dieu. Prescriptions ou recommandations dext sur Dieu. Prescriptions ou recommandations dont se voit pour le lecteur qui, le livre acheve, acceptera, nous semble-t-il, plus aisément que le texte porte sur Dreu que sur la lećn par l'auteur de Comment parler livres quel'on 'a as lus ?N'est de Coms iivesquelon ápas lus? Nest pas che sorte dimpertinente faussepiste? Tentons, pour note part, de parler un live que nous avons ly.

La question à laquelle le livre se propose de repondre prend consistance en 1940, quand Pétain devient le chef dune France en partie occupée par les troupes et les services de IAllenagne nazie. Que faire? puisser la defaite, accéter la collaboration avec au contraire, s'y opposer, mais alors de quelle façon. Le chroniqueur découvre qu'il est difficile de séloigner de a chronologie du livre qui commence par une analys . Jucien. On se souvient que Lucien Lacombe est un jeste trois Dans les quatre parties quiorganisentlensemble, rois ordres sont repérables : un ordre conceptuel qu oique, empathie, peur, de soi). À cet ordre concepluel co, enpant tuel correspond choix, se présente une décision ou

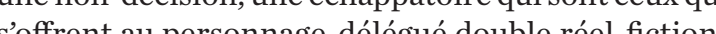
s'our d'université d'aujourd'hu Choix décision, expeur d'université daujourd hul. Choix individus écls que l'an-choixs'adossent alors à des individus réels que lauteur convoque, auxquels il se (Bon

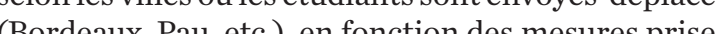
(Bor le ré an lo gnement, mise en place du STO), apparaissent dan éels : Daniel Cordier, futur secrétaire de Jean Moucin : Daniel Cordier, futur secrétaire de Jean Moude ; petite ville de Ch Gonctà venir; Les Justes peur Trocmé ct fa femme S Lousa Mendes, consu du Portugal à Bordeaux qui désobéissant as, consul nement de Salazar sione sans compter des visas a que les Juifs et tou signe sans conpter des visas afin

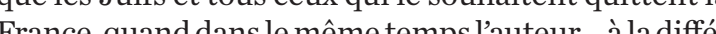
ré dirigée par l'historien vichyste Carcopino supérieure jours des Ihistorienvichysté Carcopino. Ce sont tou-

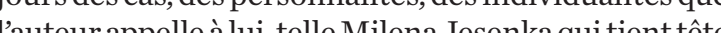
lau sonnelités camp de Ravensbrick. Comment ces perleur choix rendre compte des comportent expliquer ment la peur du risque physique masque le motif vra de ne reur faire ou à linverique masque le motif vra fait quon fare ou, àlinverse, comment se cacher du convan convaincre qu'on mène une action de résistance ? Ce ne sont pas les mesures anti-juives du régime de Vichy qui pousseront le personnage-délégué a s'engager tarhom con Und for soupcich

Une fois soupçonnées et invalidées les bonnes raisons dêtre bonnes, cest-à-direles raisons commandées parlidéologie, lappartenance à un groupe, lempathie, 
autant être capable de s'engager ou de s'approcher de action, on atteint ce que la dernière partie du livr

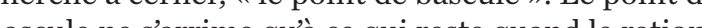
che détermo tét termes: soi-menc, les autres (et non autri,

Il faut sans doute être psychanalyste pour faire hypothèse de cette personnalité potentielle dont le sujet conscient náa nidée, nireprésentation, ni anticipation, et sans doute pas le désir chenisager ni de savoir

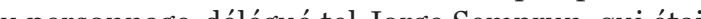

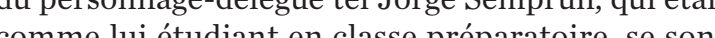
commé lui étudiant en classe préparatoire, se sont de nous, a coun

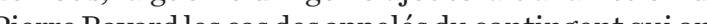
lofé comm Noël Favrelière d'abattre le prisoront re cour rabe qu'on leur confai

On se demande quel est ce Dieu, qu’on dirait volontiers athée, dont parle Pierre Bayard si ce n'est lénigm quenous sommesànous-même. Il n'est pas indifferent, ou plutot pas surprenant, que ce soit un analyste qui été celui-ci ou celui-là, résistable réponse: aurai-je chéch que la procédure bâtie pour écrire le livre paraît l'emcrorive tion quile motive. Car li y a dans lentreprise de Bayard a lais prousse ctvaité. Au sens dungenre pictura bien connu, au sens où

Michel Enaudea

\section{FUIR LE REICH, LES RÉFUGIÉS \\ JUIFS DE 1933 À 1946}

Deborah Dwork Robert Jan Van Pe

Paris, Calmann Lévy/Mémorial de la Shoah, 2012, 476 p.

e sujet du livre est le sort des Juifs qui sont parvenus à fuir l'Allemagne de 1933 à 1946. Contras'agit, le plus souvent, nullement du récit de destins de personnes rescapées, mais bien, pour reprendre la distinction de Primo Levi, de destins de personnes naudistinction de Primo Levi, de destins de personnesnaurévastés lóns dengouties (Sommersi), de vies

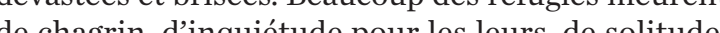
de chagrin, dinquiétude pour les leurs, de solitude. Duelques-uns, célebres ou inconnus, se suicident. Deborah Dwork et Robert Van Pelt déc sivent pleinement un aspect de la Shoah, malgré les apparence

Sans doute Fuir le Reich apprendra-t-il peu aux historiens. Mais pour le plus grand nombre des lecteurs les auteurs réunissent en un seul volume les aspects essentiels de cette histoire. Les differents moyen auxquels les nazis ont pensé pour chasser les Juifs au cours de la guerre sont soigneusement massacre au cours de la guerre sont soigneusement présentés. Reich etles réaction d'indifférence, d’im Jifs dans le Reicter ex complicité et, parfois tout de même, de résistance dan ainsi un grand nombre de mises au po live contient ainsi un grand nombre de mises au point sur les polidu Royac-Uni, de de la Suisse. Quelques figures hérrion soveétique ou de la Suisse. Quelques figures héroiques apparaissent aussi tels le consul japonais en Lituanie, Chiune Sugihara, qui dellva aux Juifs des visas de transit pour le Japon ou Paul Grininger, ch réfugiés de fa de SaintGall en Suisse qui donna aux refugiès de faux papiers (ce qui luivalut un córable jusqu'à sa mest la police et une vie miserable jusquâ sa mort en 1972). cette habitante de Zurich qui organisa à partir de cette habitante de Zurich qui organisa à partir de sa juifs arde un véritable service postal entre les réfugié juifs allenands et leurs fanilles. Le lecteur trouvera egalement dans le live beaucoup d informations sur l'histoire de IOSE, l'ouvre de secours aux enfants Lhistoire des espérances sionistes - quelques foi dęçues - et lhis Un tout le livre.

Un grand nombre d'histoires personnelles des victimes, souvent très émouvantes, viennent illustrer régulièrement l'histoire générale, que ce soit la destine de poit la destinée dillo Stefan Zweig.

On voit ainsi, dans les premières années, la France accepter les réfugiés même dans la misère. Ce n'est pas le cas de lAngleterre qui prêfere "prendre les meil- ter. Les Juifs sont « comme les animaux qui, pour se protéger, changent de couleur et s'adaptent à celle la vétation environnante. » On suit ainsi les projet à Maser Ju à Madagascar, dans la region de Lublin ou en Russie arctique. On accompagne les exilés dans tous les pays

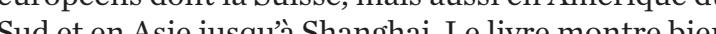
Sud et en Asie jus sua Shangha. Le livre montre bien com lles allemandes, refusent de se considérer conme une minorité et dêtre concernés par en faveur des «minorités » [p. 85].

En ce qui concerne la France, les passages sur $\mathrm{l}$ stuation des Juifs dans les camps d'internement sont accablants. Bien des récits sont déchirants comme celui

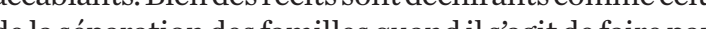
dela séparation des familles quandís sagit de faire par-

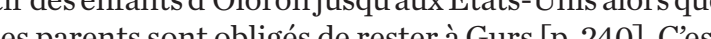
les parents sont obliges de rister à Gurs [p. 240]. Cest tir de Marseille vers les États-Unis, mais qui perd tir de Marseille vers les Etats-Unis, mais qui perdent Torch (débarquement des Américarès l Opération Torch (debarquenent des Anéricains et des Anglais Le Le livre ne sarrete pas avecla fin de la guerre etl'o voitencore des Juis denich sans compter ceux qui, de retour en Union soviétique, y sont simplement exécutés (de même que beaucoup ćn genéral Patton traiter encore, la guerre finie, les Juif «despèce de sous-hommes», son collegue Eisenhowe ainsi dailleurs que le président Truman sont ré

Le livre sachive sur les diffecttes des survivan

Le livre s'achève sur les difficultés des survivants descos erson le départ des Juifs des pays arebes en dumonde. d’Irakin Juifs à quiter un las ov Le livre est divisé de manière chepus 2500 ans.

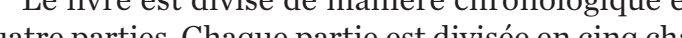
quatre parties. Chaque partie est divisée en cinq chapitres autour des mênes hines. les faits appuyés sur des dates, les homnes, les lieux, les papiers (passeports, visas, courrier, liste de rescapes), les problemes. Les cartes etsurtout les no breuses photographies histoliées au texte, très soigneusement légendées. Bref il s'esit d'un livre destiné à toute personne de bef, sogit dé l'importance de l'appareil de notes. C'est un livre clair dont la lecture facile est particulièrement utile pou ercevoir, avec la tragédie de la déportation, un aspect de l'immense quantité de souffrance provoquée par la politique des nazis et l'indifférence ou l'impuissance les témoins dans le monde.

Jean-François Forges

SOLDATS, COMBATTRE, TUER, MOURIR : PROCÉS-VERBAUX DE RÉCITS DE SOLDATS

\section{ALLEMANDS}

Sïre Neitzel. Harald Welzer

Gallimard, « NRF Essais », 2013, 640 p.

a parution en Allemagne en 2011 de ce livre coécrit par l'historien Neitzel et le psychosociologue Welzer a fait sensation. Il vient dêtre traduit pa sintéress à l'histoire de la Seconde Guerre mondiale intéresseà histoire de la Seco de Guerre mondiale (période qui n'est préci

作 peuvent être critiquées. Au départ, il y a la « chance du chercheur qui n'est jam hasard. En 2001, le professeur Neitzel tombe das rchives anglaises, sur des comptes rendus d'cos landestines de prisonic dats et hes des des armes - dan en en Grande-Bretagne. Cet espionnage de prisonniers, tainement eu lieu à toutes les époques, mais à cette tainement eu lieu à toutes les époques, mais à cette occasion il fut pratique a une échelle " industrielle » fus littéral dectint ainiré a pre l'univers de lassion : « Je fus littéralement aspire dans univers de la guerre qu se déployait devant moi.» [p. 10] Lechantillon est très ample, il sagit de conversations de milliers de prison-

Par la suite Neitzel découvrit dans les archives

Pár la suite, Neitzel découvrit dans les archives de la même façon deux fois plus importes, collectés de la même façon, deux fois plus important. Au total, ce furent donc près de cent cinquante mille pages de trans de a cas de consersations authentiques, de 
collaboration avec son collègue de l'Université de Giessen, Harald Welzer qui jouissait déjà d'une grande otoriete pour ses travaux antérieurs. On peut consiperer, avec les antenrs, que les prisonniers n'etaient pascon ix cent. Le res six cents pages. Les auteurs procédent dabord a une lly port II $^{\mathrm{e}}$ Rei (chapion plus an plus long, aborde en une cinquantaine de rubriques tour qui la guere est fie et qui se lassent aller: pour qui la guer est finie et qui se laissent aller : ils on'est la guerre, par exemple celui de pouvoi suprême quest la guerre, par exenpleceluide pouvoir nitrailler des civils en fuite quand on est aviateur, de couler

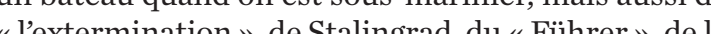

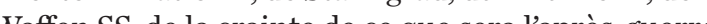
Waffen SS, de la crainte de ce que sera laprès-guerre. La conclusion de louvrage contient quant à elle des . fascination. L'horreur est docume touche d'authenticité.

Les exécuteurs, les bourreaux, parlent à leur alter ego sans aucune retenue et on peut le supposer, sans dissimulation, comme ce caporal qui a exterBelgique en 1940. Ils n'expriment évidemment pas oind en moind regret. Les soldats qui sont allés sur le front par eux-mêmes ou dont ils ont été les témoins. Sur « parternlextermination » (les auteurs, pour des raisons peu cón con úm de ces « tenoins oculaires» est presque insoutenable. ins la var scies timable: on apprend par exemple que les exécuteurs touchaient une prime. Comme lecrivent les auteurs

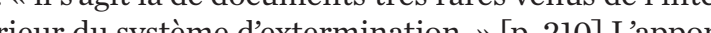
rieur du système dextermination. » [p. 210] L'apport decisitutive pour la recherche est de prouver, par déclarations prononées devant des enqûteus ou un tribunl qu'un très grand no des enquêteurs ou tex mands savaient que les Juifs étaient exterminés dan Timothy Suder) Reppelons que dix-sept million de Tho hy Snyder). Rappelons que dix-sept milhions de 1945. Citons les auteurs :
Pour résumer, on peut dire que l'extermination des Juifs tunélément de l'univers cognitif des soldats, et ce, dans une bien plus grande mesure que ne permettraient de lattendre s études récentes sur le sujet. Il ne fait aucun doute que ous ne savaient pas tout, mais les procès-verbaux découte revelentent tous les détails de l'extermination, jusqưaux mises à ort par moxydedecarbone foule de to ra ation, raison pour lacullo onpent aussiconsider, dansce tués. [p. 180]

Par ailleurs, il est remarquable que, sans doute par solidarité de situation, beaucoup de ces prisonniers solidarite de situation, beaucoup de ces prisonniers armée aux priquaient le sort atroce réservé par leu amée aux prisonniers de lAxmée rouge. Cependant, un un tès prudo dan lair du temps. Les auteurs sont certes très prudents, mais ils ne résistent pas toujours à la cour souhaiter (tante plation de la violence guerrière ». Sur cette question et sur la rem dautres que pose le volume, nous renvoyons à Coquio sur le site fabula org [juin-juillet 2013 , vol. 14 no 5 ] : C'était des autetaitpente historiqu la pécente les historique la plus récente les conduit a proposer de arguments parfois douteux, comme, par exemple, delextermination des Juifs par Himmler levocation delext cours de Posen «qu'elle n'etait pas le centre menta 190] Ouccupations des soldats mi mêne de la SS. » [p. 190 Ou encore cette fâcheuse tautologie: «la majorite toutefois es téc du terme, Nia), du terme, Nda), mais celle de la guerre. » [p. 501] Et "pour quicon'ils aient mené, de leur points, on ne peut "gas dire qu ils aient mené, de leur point de vue, n [p. 356] Lexpression «deleur point gueverat. [p.356] Lexpression «deleur point de vue» est le pivo de largumentation: a la limite, les soldats de la Wehrmai qui ont participe à des massacres de Juifs n’étaient pas et Welzer insistent beoucoup sur la dinension de guerre comme « travail» pour les soldats, ce qui contribue fortement à cette " désidéologisation ». Au fond e repro est d'avoir privécié l'approche socio psychologique est davoir privilegie lapproche socio psychologique au détriment de lapproche historique. La définition du u justifier de "partisans » qui a servià ordonner et à « justifier » les ma (Oras sur le front de lEst et en nurope orcidentalé(Oradour, les Fosses Ardéatines) n'est jamais proposée par les auteurs, alors même qu d'êtrelà Lelivre ses victimes qui avaienten le seul tort detrela. Lelivesen, sur ce point, Ignorer le remarquable traval de mónoire mené par Hannes Heer et sonequipe dan rantes les chich pas cité dans la bibliographie. Limpasse est aussi faite idéolog cos des crimue quia faitdebeaucoup soldats allemands des crinine de qu concerne la traduction, on peut contester le choix de "ethnie » pour traduire Volk, il ausit fallu conserver "peuple », plus en accord avec lickologie nazie. Mais, encer

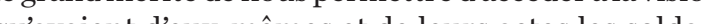
allemands de la Seconde Guerre mondiale. Robert Kahn ignorance cynique du passé. » C'est donc entre deux cueils, entre la sidération devant un passé terrible et la fausse conscience de ce passé refoulé, que créateur et pectateur doivent passer: mais l'art devrait permettre d'échapper au dilemme.

C'estlà précisément que se situe l'ouvrage collectif dirigé par Alain Kleinberger et Philippe Mesnard, qu propose un très riche panorama de ces « représentaton a tour d'Europe? Dans quels pays? Avec quelle restion? Qurope Dans quels pays? Avec quelle réception?

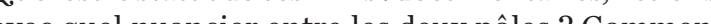

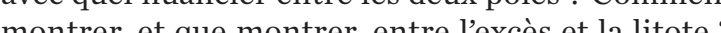
Telles sont quelques-unes des questionsquelouvage permet de poser.

es auteurs sont partis du constat que les fiction sur la Shoah se multiplient, surtout au cinema, à un

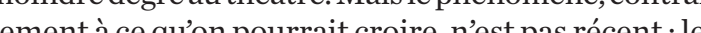
renent a ce qu'on pourrait croire, n'est pas récent; les de la guerre. Dans cette première génération de films, Ariel Schweitzer atle précé genér (1947) un des premiers films israéliens dur la Shoah, à fis docén a fois documentaire et fictionnel. L'interêt de ce film (1961) le cinéma israélien tend à ignorocès Lichman (1961), lecinema israelien tendàign lui-mên int utilisé des extraits de ce film comme imaes d' ont utilisé des extraits de ce film comme images d'ar-

Au même moment Wanda Jkubow

Aüméne moment, Wanda Jásowska tourne $L a$ Derniere Étape (1947-1948), analysé par Alain Kleinberà. Jirk à Birkenau en 1943. Le film, tourné à Birkenau, avec pour figurantes danciennes déportées, est critiqué par

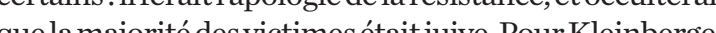
que lamajorité des victimesétaitjuive. Pour Kleinberge tann vient d'ailleurs, car après-guer. Mais loccultation vient d'ailleurs, car après-guerre en Pologne, «les victimes étaient, rétrospectivement [...] considerees comme polonaises. $\gg$ Message également quelque pe Frank Stern: Staudte (1946) pose bien la question Staudte (1946) pose bien la question de la culpabilité allemande, mais « coupable de quoi, au juste? » Néanmoins, quelques-uns des meilleurs films representan nés à fract 'égitime, et jusqu’à quel point? Omer Bartov semble admirablement pointer le dilemme, quand, a propos lim (1994), ilécrit que cefim est implact exp à a fois les efets destructeus de la expose a la fois les effets destructeurs de la mémoire et de la conscience contemporaines causée par une 
d'expérience cinématographique, de technologie, de concentration artistique et de mauvaise conscience. Du coté français, Antoine de Baecque interroge God a la Shoa. Godard refuse la representa Gothétiqu la she le mo re esthétique, cherche le montage juste " qui permet de representer charchear lexternination. Ses choixmilipest palestinien en 1969-o sur les pouvoirs de lart: Histoire (s) du cinéma stig. la nonté des perils, et lui-mêne se juge coupable:

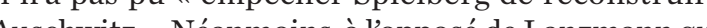
che l'archive l'image d'époque (inexistante ou refuse larchive, limage dépoque (inexistante ou truquée), Godard insiste sur la présence des images de ition bies sonch le la tragediedusiecle. L'oppode Notremusique nest loncpas une de Notre musique n'est doncpas uniquenent politique, ou, pour mienx dire, ellétiques. Ce qui est dévelion politique des choix esthétiques. Ce qui est développé a a la rorly (quive rôle de la résistance politique à Auschwitz) et de Rémy Besson qui synthétise le genre de Shoah en repren

Cette question traverse, peu ou prou, tous le Certes, puisqu'il sagit, comme le formule JeanMichel Frodon, de «La Shoah conne discriminant

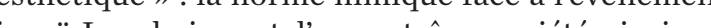
inoui. Les choix sont dune extreme variéte, ainsi que

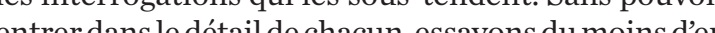
entrer dans le détail dechan, ess yons dus poins

Ener quelques lignes de force.

En prenier lies, le documentaire est-il un « document » neutre ? Luba Jurgenson démonte, ave lexemple de Theresienstadt, les mecanismes de propur commande des nazis. Philippe Mesnard explore sur commande des nazis. Philippe Mesnard explore la «zone grise » (Primo Levi) des Sonderkommandos, our ces de la lutte armée, aux résistapposition aux héros de la lutte armée, aux résistants. Annette

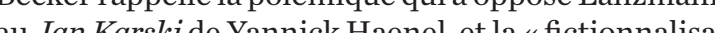
tion tion» du personnage historique: «L héroíque courrie catholique de la résistance polonaise s'est transforme catastrophejuive, en Justeparilos nations, et en quasi-Juif. » Il ressort de ces trois articles que le documentaire n'est pas plus « objectif» qu'une fiction mais procède aussi de choix esthétiques et politiques. En second lieu, ce qu’on pourrait appeler «les défeinle monstre conment doun un proces, Matthias Steinle montre comment, dans un processus d'autovictinisation, les docudrames allemands deplacent

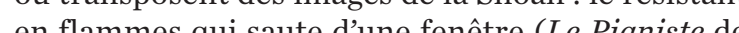
en flammes qui saute dune fenêtre (Le Pianiste de une équi cstransposédans Dresden (2006), suggérant une équivalence entre le bombardement de Dresde et le ghocid

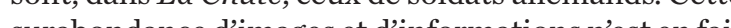
surabondance din que le symptôme et le moyen d'une nouvelle phase de

Pour l'Italie, Millicent Marcus rappelle que, dans la mesure où $80 \%$ des Juifs italiens furent sauvés, l'Italie évite de se confronter au rôle qu'elle joua dans lHolocauste, alors que les lois raciales de 1938 sont une initiative de Mussolini et non un diktat d'Hitler. Or lecinénaitalien, pourtant tres engagé sur des sujets sociaux, a tardé á s'enparer du sujet. Lauteur suggère une interprétation incess siondellonssethe nouvelle façon de penser le phénomène fascistea parti de la perspective juive et, dautre part, lantux dimmigrants du Tiers monde obligerait à poser la question la tolérance à l'autre.

Pour la France, comme pour l'Italie, le sujet a été longtemps occulté. (Il faut mettre à part Nuit et brouillard, auquel plusieurs auteurs font allusion, mais qui reste un phénomène isolé à sa date.) Ce n'est que dans les années 1970, après Le Chagrin et la pitie (1971), que le cinéma de fiction commence à s'y attacher Anny Dayan-Rosenmann inscrit Monsieur Klein (film français de Joseph Losey), dans la lignee des films de annés 1970 quicommencent en France, conme Les Guichets du Louvre ou Lacombe Lucien. Lauteur soulignelaveuglement du protagoniste, qui ne « voit » rien alors que le film nous montre les préparatifs de la rafle du Vel d'Hiv. Pour la Hongrie, du románonyme d'Imre Kertészans destin, adapté du roman éponyme d'Imre Kertész avec la collaboration delauteur,et dont la réception négative contraste avec le succès du roman; elle suggère de replacer le film dans lhistoire du genre, et voit un « symptôme pays. En transweritén le passionnant article d'Omer Bartov, «Le "Juif" au cinéma: du Golem à Don't touch my holocaust », étudie dese Cens : son analyse de Hamsin de Daniel Wachsma (Isräl, 1982) me paradoxal, des stéréotypes sur le Juif et l'Arabe.

En troisième lieu, les stylisations artistiques. En troisieme lieu, les stylisations artistiques. Vicaire de Rolf par vicaire de Rolf Hochhuth et son adaptation filmique,

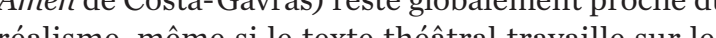
imites duc dides du genre : insertions de docunents, longues didascalies, intervention qui séparent Hochn. dh du " theatre épique ». Se pose 1963 nest la question du public: les spectateurs a forger un à forger une inage, une conscience En revanche, les deux autes artes con se differencient nettenent de la représentation, qu' slisse du travall de Sarah Kane sur le traumatisme (Elisabeth Angel Perez) on du (theatre defigies de Meister) qui, par la manipulation de figunes dans une mécan du camp, anòne les acteurs à entrer dans le mécanisme psychologique du fonctionnement du

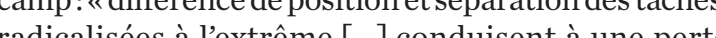
to totale du sens moral des actions. » En rester la serait evisenit comme le formule Walter Benjameurs, mais il s'agit, comme le formule Walter Benjamin, d'arracher limage au continuum de lhistoire universelle, seule condition dun regard critique. " faut jouer à » et non pas s sidentifier.

Dans tous les articles, se pose, implicitement ou non, la question de la réception, laquelle renvoie son tour à celle des intentions de lauteur. Christian Bret, devant la pièce Anathène, de la compagnie belge Groupov (Avignon, 2005), voit Auschwitz, cela contre de même, lisant le roman de Philip Roth Nemesis, ily de mêne, lisant le roman de Philp Roth Nemesis, il y voitune allegorie du camp, commeletait La Peste. Dan es deux cas, sa uvision » est convaincante, et rappelle que lintention de lauteur - longtemps abandonnée par cost qu'un paramètre parmi d'autres de la reto

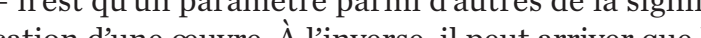
the défendue par un auteur soit rejetée que

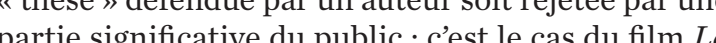
Question humaine de Nicolas Klotz (2007), analysé par Stéphane Bou. Ce film, adaptation du roman éponyme de François Emmanuel, s'inspire notamment d Modernite et holocauste de Zygmunt Bauman, qui étabit formules-chocs, comme "Personne n’ose dire que 'Amtsprache d'Adolf Eich lamtsprache dAdolf Eichmann est très proche de la de l'or

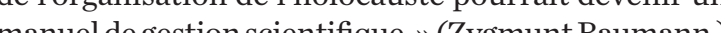
Ce film, tì

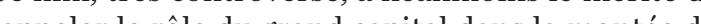
rappeler le rôle du grand capital dans la montée du

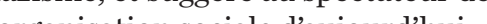
Lor

La controverse a également fait rage autour de $L a$ Vie est belled. Benigni.une partie du publicn'acceptait pas cette vision ludique et onirique du camp. En marge Susan Rubin Sur Inglourious Basterds de Tarantino, Susan Rubin Suleiman s'intéresse au film de Benign, comme à Train de vie de Minalleanu : ce type de film toire telle quelle aurait pu être, et quic cul de lhistoire telle qu'elle aurait pu être, et qui coexiste avec la connaissance tragique de ce quelle a été. »Ces repréentions cont pas de nature à encourager le négationnisme: au histoire, c'un ciné que put d'istore qu'un cinéaste peut se permettre « des écarts réel tout in $[\ldots .$.$] quirenforcent notre connaissance du$ belle réflexion nous incite à envisager l'histoire et politiqu Anne Roche 
comme le fit déjà Tzvetan Todorov (cf. Les Abus de la mémoire, Paris, Arleà, 1995) - sur les différentes expressions des dérives de la mémoire.

Après une brève introduction sur le rapport entre la mémoire et 'histoire- rapport qui n’a pas toujours été compris et interprète correctement dans les célébrations officielles - lauteure choisit de s'attarder sur trois le discours public : la négation, la la mémoire dans tiscours public: la négation, la sacralisation et la tis tincts dont ellemontre tout for les connections: «Ces trois abus s'enboitent connme les pièces d'un puzzle, (c) es protagonistes rendent compte.

En résulte une mémoire collective (qui ne concern pas seulement l'Italie) « exposée aux feux croisés » de facteurs de formation et d information aux motivation diverses, dont laction suscite, en général, des controverses, des conflits ou des déformations de l'histoire (sans parler dé la mémoire). Etcest aussila raison pour en rello en renonçant à approfondir (du moins dans le débat pub́c et lans lusage courant) le rapport entre notre

présent et notre histoire, nationale et européenne.

La reconstruction de ces trois formes majeures de dérives de la mémoire se présente conme une reconaissance synthetisce des dinesrestes étapes qui ont

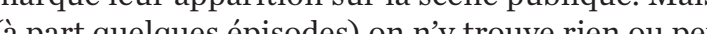
(á part quelques épisodes) on n'y trouve rien ou peu sur le débat critique que ces etapes ont provoque, pa exemple chez Primo Levi (pourtant cite), ou sur les extes histor ituries, ences ont accompagné une réfexion critique sur les ouvrage des auteurs du négationnisme, de la sacralisation et de la banalisation. On n'y trouve de mêne aucune trace ont généré par opposition des nouvelles études et de . nouveaux acquis, par exemple en matiere de fonctionlogiqu "sur les sites du génocide. Ens

En conclusion, louvre de Valentina Pisanty est un bon outil de vulgarisation. Mais, contrairement à certains de ses autres ouvrages, le texte n'offre pas deléments dont

\section{MEIN KAMPF, \\ HISTOIRE D'UN LIVRE}

Antoine Vitkine

Paris, Flammarion, «Champs histoire », 2013, 332 p.

originalité de l'étude d'Antoine Vitkine est de s'intéresser au livre Mein Kampf en tant que tel,

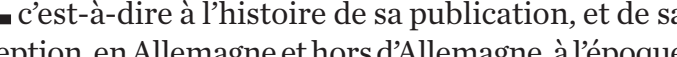

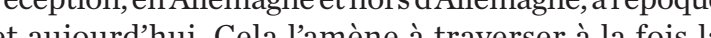
to d'Hitla laméne à traverser à la fois la bogrante du lazisse la seconde Gne nours sous langle des effets de ce livre qui n'est pas comme les (qui reprennen articulier l'antisemitisme) que par son impact.

L'auteur étudie d'abord les ventes du livre, imporantes dès avant la prise de pouvoir, et son accueil en 列 mépris mepris. Aucun groupe, aucun parti ne semble prendre la mesuré le levenent, que ce soit le parti communiste,les Ĺghises, ou mêmelapressejuive. Maisen 1933 quand Hitler devient chancelier, un million d'Allemands achètent Mein Kampf, avant même que l'Etat lo lance une canpagne de propagande. Les mairie loffrent auxjeunes mariés; le partinational-socialiste mais a ou l'offrent. L'endoctrinement de lajeunesse étant une priorite des nazis, on publie des livres d'images qui en À l'es extraits, des céts de la vie de Hitler.

Al'étranger, après 1933, le livre est traduit, mais dan des versions expurgées de ce qui touche à la politique étrangère. « Sij’avais pu deviner en 1924 qu’un jour je deviendrais chancelier, jamais je n’aurais écrit Mein Kampf » aurait confié Hitler à un proche: le texte dévoile le gêner du moins tant que le rapport de forces neu le gêner, du moins tant que le rapport de forces n'est pas en faveur de lAllemagne. Il veut donc restreindre laccès des pays étrangers a son texte. Ceux-cis'alignent parfois sur les désirs de lediteur allemand: c'est ains quaux USA et en Grande-Bretagne, leséditeurs publien En une version censuree par Eher-Verlag.

En 1933, l'Italie mussolinienne achète fort cher étant juif,Bompiani fait disparaître son nom. Les moti- vations de l'éditeur sont obscures: s'agit-il d'avertir les Italiens d'un danger, ou de les endoctriner? La même ambiguïté se retrouvera en France.

En effet, la France est particulièrement attaqué dans Mein Kampf.Or Hitlerveut rassurer l'opinion frança . de Brino (for firme pacifiste et minimise Mein Kampf, «un livre plein dimprécations écrites en prison. » Et il refuse looff́

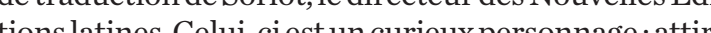
tionslatines. Celui-ciestun curieux personnage: attire

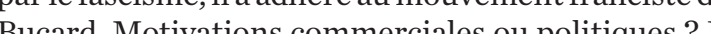

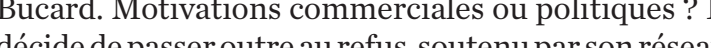
décide de passer outre a refus, sontenu par son résea au ministere d'Anciens Combattants. Au passage, Vitde Lyautey éć ryatey, "Tout Français doit lire ce tive ", qui a après le procis, la Confédération des co tribuabs, pris le procès, la Con tés à cho de contribuables, puissante association très à droite, patronnée par

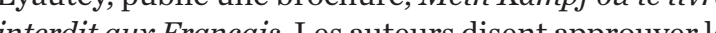

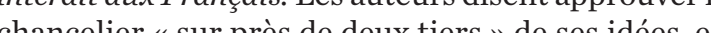
chancelier an pros de deux tiers ne ses idées, en particulier franc-maçonnerie, la presse. Et Lyauteyles en felicite... ce qui donne à sa phrase une certaine ambiguité, et $p$

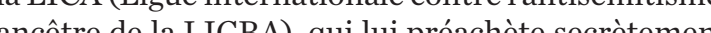
ance cinq mille exemplaires. Ces exemplaires sont envoyés à des parléné pos, André Calmettes, lun des traducteurs, témoigne: Nos députés n'ont pas le temps de lire les exemplair. cos députés n'ont pas le tenps de lire les exenplaires que nous retrouvons en grand nombre chez les bou-

quinistes sur les quais.» [Témoignage amilial, inédit] Hitler porte plainte, la Société des gens de lettres sassocie a sa plainte. Lors du procès, en juin 1934 lavocat de Sorlot souligne lintéret de faire connaits crace du chancelier et notanment sa haine de très relayé dunal ne le suit pas. Mais linterdiction, très relayée dans la presse, accroit lintèrét pour le livre. Sorlot publie une brochure dextraits, portant un bandeau "Le livre interdit aux Français » et contreattaque « Les droits dauteur de Hitler? Mais qu sest donné le ridicule de songer aux droits d'auteur brûlé publiquement les ouvrages? brûlé publiquement les ouvrages? » L'argument est

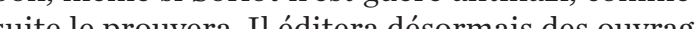

favorables à Pétain et à la collaboration, mais continuera à vendre Mein Kampf clandestinement, ce qui lui servira à la Libération lors de son procès : d'Astie de la Vigerie témoignera qưil « a fait son devoir de Françis en servant à éclairer la Résistance en livrant es exemplaires de la bible nazie.

Comment les Français reçoivent-ils cette lecture, intégrale ou en extraits ? On est frappé, à près de quatre-vingts ans de distance, de l'inadéquation des reactions. Les politiques pensent qu'il s'agit d'un livre voit vén volonté de puissance prussienne. Certains pensent cequin'est po pour leur déplaire. L'aveugla courtast pánéral Dès décembre 1933. Trontsi, vourtant pas général. Dès décenbre 1933, Trotski, qu deHitler, et démonte son prétendu pacifisme, dalition journel Lórité, dontlaudiencest journâla Verité,dont laudience est surtout militante. Suarès dac $L$ Revue littéraire, mais ils no n Hitler, Eher-Verlag, fit pulie Ma Doctrine, chez Fayard Il rerit d'une sorte de « versio Doctrine, chez Mein Kampf rédigée par deux journalistes qui toutes garanties : l'un est à Candide, l'autre à Je suis partout! Le texte est toujours antisém lautre à Je suis

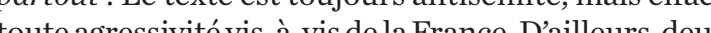
toute agressivité vis-à-vis de la France. Dailleurs, deux mois apres Munich, le 6 décembre, est signé un accor Aveclaguere, l'intét pour

Avec laguerre, linterêtetpour Mein Kampfs' intens que Certains sont des mises en garde, d'autres, comme les Értains sont des mises en garde, dautres, comme les

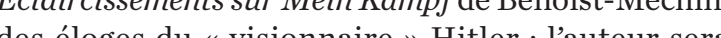
Es conce à mortà la Liberation.

Après la Nuremberg, le livre est utilisé comme preuve à charge. Mais les Allemands, qui l'ont presque tous possédé, lant-ínégation qui se meten en place dès 1945. [Cf L Larticle d'Ingeborg Rabenstein-Michel, «Adolf Hitlers Mein Kampf or Rabentein-Michel, « Adolf Hitlers Mein Kampf, gezeichnete Erinnerungen an eine große Zeit veur par et veur par et dans la BD », in Témoigner entre histoire et mémoire, $\left.\mathrm{n}^{\circ} 113\right]$. Ce qui explique en partie qu'il y aitpe 
diverses formes, qui ont «imprégné » l'ensemble de la population, même sans une lecture intégrale du livre certes, et lauteur le soullgne, licéologie antisémite qui a prepare la destruction des Jif deur trouvait pas uniquement dans Mein Kampf. Mais le la fón la a four leurpelle à justetitre les limites de la Tán.)

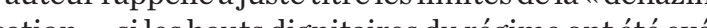
cús a Nules hauts digntaires du régine ontété cutés à Nuré ans le privé ou dans la politiqu. Ains, le secrétaire destrate

L'autenr exam d'Allemagne, après la guerre. Hitler étant resident right du live pour l'Allemagne entiè reç le copyd'en interdire la diffusion, y compris à l'et la mission en interire la diffusion, y compris à letranger, car les siturions sont tìs diveres.

Dans soins pars, Mein

Dans certains pays, Mein Kampf est interdit, au mons en théorie. L'Etat de Bavière, régulièrement inire retirer le live de la vente en Russe, au Porsugal, Répliqu en République tchèque, en Pologne, en Turquie; pour tions ontété publiées ces dernières années, le livre est (lons ontéte publéesces denières an nobjet-culte de lextreme droite.

Dans d'autres pays, lachat du livre est légal : en Italie, aux USA, en Angleterre. En France égalemen alors que beaucoup croient encore qu'il est interdit. droite, r compris nationniste (Rassinier) et cêm. droite, y compris négationniste (Rassinier) et contiì 1960, ouver après 1960, ouvertement. En 1978, dans un contexte de de de Darquier de Pellepoix), la LICRA porte plainte pou faire interdire Mein Kampf, alors quelle avait paye pou diction, mis exige l'encart d'un avertise pas dinterdiction, nais exige lencart d'ú avertissement dan chaque exenplaire. Fayard prépare une édition crtique pour 2016

En Allemagne, le livre reste interdit, sauf aux cher列 publication dextraits commentés, dans un but scien-

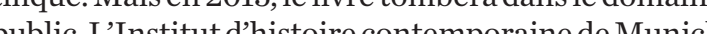

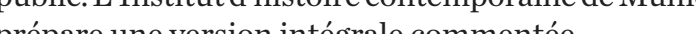

Hors d'Europe, « dans des pays où le nationalism s'exprime sans la retenue que donne l'expérience de l'Histoire » (comme le dit gentiment l'auteur), Mein Kampf a un succès populaire : en Inde, en Asie, en Afrique francophone, dans le monde arabe. L'auteur rappelle que dans les années trente, le nationalisme arabe et le nazisme ont des ennemis communs: :'AngleSion sont un bestSion sont un best-seller, loin devant Mein Kampf, ce dernier se vend toujours dans le monde arabo-musulman, surtout sous' forme enquête de de lecteute par lauteur Turqueno de lecteurs lont réllement lu, is sy rérent, de façon contexte de leur vision du Antoélo-palestinien.

Antoine Vitkine a défini son travail, dans une interview, en termes modestes : « Ce n'est pas un livre dhistorien a proprement parler, plutôt un livre dejourdit dit, sur des sources allemandes et françaises, au premier chef lenquête din einese Buches : Mein Kampf 1922-1945 (Oldenbur. 2006), la thèse de Josselin Bordat. Mais son ouvrage a le mérite de mettre ces informations a la disposition du public, de façon claire et argumenté. Et il prend parti dans le déba dire Mein Kampf mais le décoder, compras interdire Mein Kampf, mais le décoder, comprendre le conditions historiques de son apparition. Et en même temps, savoir que histoire ne se répète pas, ne pas faire capables tronter capable deconprendre lanouveautédunévenement.

peut quêtre d'accord avec ce programme.I

$$
\text { Anne Roche }
$$

\section{HITLER ET LES PROFESSEURS}

Max Weinreich, Samuel D. Kassow, Martin Gilbert (Préface), Isabelle Rozenbaumas (Trad.) Paris, Belles Lettres, 2013, $393 \mathrm{p}$. ax Weinreich ? À scruter les index biblioaphiques d'ouvrages qui ont connu (et tomes de La Destruction des Juifs d'Europe de Raul Hil- berg, ou qui ont nourri la controverse, tels l'ouvrage de Daniel Jonah Goldhagen, Les Bourreaux volontaires de Hitler, et, plus récemment, le livre de Christian Ingrao, Croire et détruire - dont le sous-titre est Les intellectuels dans la machine de guerre $S S-$, la tentation est forte de répondre : personne ou presque, tant brille par son absence le nom de Max Weinreich. Pourtant quand Raul Hilberg commence en 1948 ses travaux de recherches qui lui permettront de publier en 1985 aux États-Unis, sa somme, le livre de Max Weinreich est paru depuis deux ans à New York, en yiddish est paru depuis deux ans a New York, en yiddish
Hitlers profesorn - et en anglais - Hitler's Professors - traduit selon toute vraisemblance par l'auteur luimême du yiddish en anglais. Aussi la publication de ene divre yition en

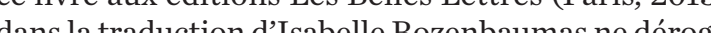
dans la traduction d Isabelle Rozentaunas ne dèroge pas au caractere savant sinonéndit qui est la marque

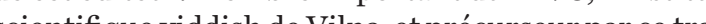
sich vall de bien des rection du national-socialisme, on peut penser, a priori, mais car depur pus rescapés ne sont pas restés « les bras croisés ».

Les quelques noms familiers en France d'intellecuels ou de docteurs d'Université compromis avec une idéo cou docteurs d Univén idéologie en train de conquérir les esprits et les masses - Junger, Carl Schnitt, Heidegger - disparaissentensedo teurs Zélés gen de savoir ils mières. Zéés gens de savon, lls sactivent, dès les pre-

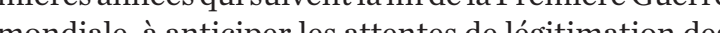
thèses rampantes du nazisme. Weinreich montre bien compantes du nazisme. Weinreich montre

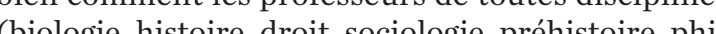
(biologie, histoire, droit, sociologie, prehistoire, philosophie) font sans hésitation le décisif premier pas enction rière ou affinité idéologique, ou les deux réunis cer iere ou à ne compte guère. Compte ce qui en rèsulte : à partir de 1933 '’ stupéfiante proliferation dinstituts, de centre détudes et de documentations, de fondations à dévels à l'étude de la question juive, à fes races. à développer létude à la question juive, à fabriquer une science anti-juive, à étudier l'influence juive su "la vie ecclestastique en Allemagne ». On n'en finit pas de recenser ces lieux, ces tribunes où se prépar de race, d'espace, d'espace vital, de sang, de Juif et qui vont nourrir le programme de conquêtes des territoires l'Est du Reich et les effroyables menées exterminatrices qui les accompagnent

Max Weinreich ne problématise pas, au sens où on lentend aujourd'hui, la documentation qu'il a rassemles faits » est son mot d'ordre. Les faits ce sont bien

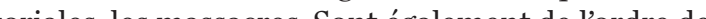
its les directives instruction 列 le jor de l'authenticín sommedisait le jargondelauthes (a) esinterto ensure d'orencher. dorganismesproducteus de rapports; jamais assez de débusquer, traquer à l'insu même du plus aryen des chos hommes, ce qui est juif. Il y a les textes, les faits et pa le Francort en mars 1941, les congres jusquen 1944 de

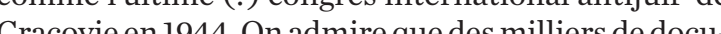
Crové

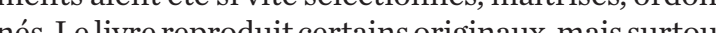
Wein. Livich reproduit certains originaux, mais surtout et références. Iln'écrit pas don la langue ses sources mis dan celle des victimes, langue parlée lue, danses

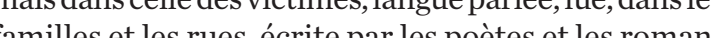
familles et les rues, écrite par les poètes et les romanVilna (Vilnius. Latugue particulièrement àlhonneur Vilna (Vilnus, Lituanie) avant Iinvasion nazie, langue qúléb

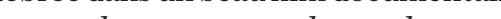
Ceuple pour un peuple sans langue.

Cest cela que dit en presque dernière phrase de son livre, au bout d'un travail mû par la colère et la protes-
tation furieuse, l'arpenteur Max Weinreich :

Àsupposer que l’on plaçât les individus assassinés en ordre de marche, la file de squelettes s’étendrait de New York a San Francisco, puis encore une fois de San Francisco jamais ces victimes de «'Ordre nouveau» pouvaient Jementjeter un regard sur l'Europe de l'après-guenteu-

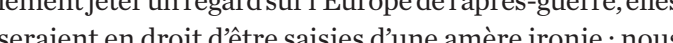
qui étions, parât-il, la causse de tous les mere ironie : nou quictions, parait-il, lacasedetors les mauxne sommes plus là, pourquoi n'y a-t-il toujours pas de quiétude? 


\section{NOUVELLES PERSPECTIVES \\ SUR LA SHOAH}

van Jablonka, Annette Wieviorka

Paris, Presses univers, 2013, 128 p.

La Vie des Idées », 2013, 128 p.

e champ d'études qu'est la Shoah est abordé et traité de différentes manières du fait d'une multi- tude de sources, de questionnements et de formes verce dine re Cost est justement lobjet de cet ouvage coordonné par ' preser raphie et d mpliquent.

Annette Wieviorka installe le cadre de ces nouvelle cherches en énumérant trois verbes très évocateur pour la mènoire : comprendre, témoigner et écrire. lifier l'histoire juive, tristement célèbre espour quale vérita le vértable boom de la mémoire de la Shoah »[p. 6] écir pions des re à pionniers des recherches, à savoir Raul Hilberg et

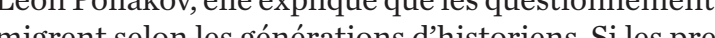
miers travaux restent confidentiels, l'ous. Si les prearchives en 1997 a multiplié les études et leur pe

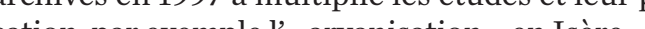
cation, par exenple l" aryanisation » en Isère.

Anette Wieviorka insiste aussi sur le fait que les historien historiens, puisque ce sont eux qui ont entrepris des

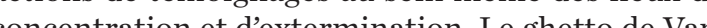
covie en est un exemple. Une étude est cepend sovie en est un exemple. Une étude est cependant (1998-2008), puisqu'ily créele d'uatiśm d'untisén une histoirécrite de tous les points de vue: celui des dilemands, ditler aux Allemands ordinaires, celu es soctés dins lesques .Cest la mise en parallele de deux historiographies.
Toutes les études sont désormais possibles pour es nouveaux historiens puisqu'ils bénéficient des trales nouveaux historiens puisqu'ils bénéficient des travaux de leurs annés défricheurs, d'une large ouverture darchiveset de la possibilite dallerconsulter temoin et archives a lautre bout du monde. De ce fait, on trouve de plus en plus de quêtes familiales sur la vie de mentring

intéressé aux archive nazies en Allemagne, dans la ville de Bad Arolsen. C'est là que se trouve le Service international de recherche ner le travail de rechen creée en 1948, devait termiet de ré de la de la guerre. Lobjectif premier de ce service etait une priorite donée aux recherches des familles et non aux de l'inces. Existantencore aujourd hui, «elle témoigne de linessante quête de mémorre, du travail de deuil jápais interis réparations allemandes. » [p. 19] Les activités du SIR sont nombreuses. recensenent des tombes et fosse tion d'ares, dédonmagements des victimes, classificationd a archives (on ne comptabilisepas noins de 17 millions de documents : du SIR sont ouvertes à la recherche depuis 2008 . Si les documents n'ouvriront probablepermettro de nouvear chanps dinvestigation, ils perinettront cependant de completer les recherche

Tal Bruttmann s'est penché sur la Shoah dans les bureaux et, plus précisément, l'application de la politique antisémite des administrations sous Vichy. Il sagit de docrimintion a instructions, à un degré ou à un autre, la discrimination: instructions, correspondances entre services ainsi que des formulaires. «Chaque adminisà intégrer l'antisémitismes [p.41] En été amenée à intégrer l'antisémitisme. » [p. 41] En effet, aucun

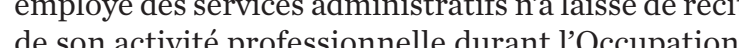
de son activité professionnelle durant loccupation. Cette integration va même, en 1941, jusqu'à l'apparition jur un forle-ci est parfois distinguée dantesesé est-il juires .es études pan dis laires. Les études concernent icitous les échelons de la juride administrative. Les documentsidentifiant les Juifs sont devenus le principal instrument de leu arestation. " Ressource de lhistorien et enjeux de la mémoire juive, linstrument bureaucratique apparaît

La méthode quantitative pour la communauté juive de Lens est le sujet traité par Ivan Jablonka. Trois moments ont permis d'identifier et de quantifier les Juifs: le recensement de la fin 1940 (par auto-déclaration), l'aryanisation des biens et la rafle du 11 septembre 1942. C'est par ce biais que le processus d'extermination commence et la logique qui la suit aussi, à savo « Un Juif est un Juif, et le sentiment patriotique et l'intégration n'y changent rien. » [p. 56]

L'étude d'Audrey Kichelewski porte sur un ouvrage traitant des relations polono-juives, dans lesquelles les traitant des relations polono-juives, dans lesquelles les preniers auraient participé aux crines des seconds avec comme motivation principale lappât du gain. L'appropration des biens conce des la phase Ce Ce sont «les moissons macabres» [p. 63]. Pour Emajuels pour rendre possible ce type de comportement juifs pour rendre possible ce type de comportement ultra-violent à leur égard. Cependant, la causalité économique de ces actes est relative puisque les Allemanis sétaient, au préloble, déjà servis. Daprès des archives, Cest la campagne moyenne qui a pris part au toriographe de toriographie de la Pologne communiste. Pour qualifie ces actes, on utilise le terme de "chasse aux Juifs

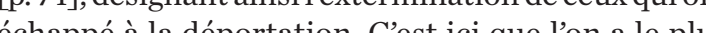
échappé à la déportation. Cest ici que lon a le plus besoin du concours de la population locale polonaise. Enfin, un tabou deneure à ce jour : lassourdissant silence de Lusise pol sysentiment à ce qui se passait.

Pour Florence Heymann, les ghettos auraient peuttre été des lieux de résistance juive. Pour autant, ghetto representait une étape préparatoire à la Solution finale. Aussí la vie qui règne à l'intérieur de ce Jifs, au cón juifs, au degré dauto-admínistration, à la solidarité (ou a sour absené Ka ta diférentes stratégies de survie. ghettos ne seraint pas soulevés, car « ghettos ne se seraient pas soulevés, car «ils savaient qu'ils ne pourraient pas survivre longtemps dans les Ivan Jablonk Ivan Jablonka conclut cet ouvrage en evoquan insi, la Ainsi, la recherche historique empêche la mémoire de senfermer sur elle-même et de se racornir. De plus, e ces temps de relativisme moral, «1Holocauste sert boussole. C'est un absolu, un absolu du mal. » [p. 93 ] l'obnubilation, l'anti-intellectualisme, la banalisation, a fermeture et la bonne conscience morale. Cela soulignerait ainsi le caractère artificiel et moralisateur du devoir de mémoire, soit la transformation du souveni en obligation. Pourtant, le renouvellement de l'histoiographie nourrit une autre manière de se souveni. Le seul devoir qui s'impose à tous est celui de la vérité. Cest pourquoi un tout nouveau travail est porté sur mémoire, et celui-ci provient des « petits-enfants [p. 102].

Les réflexions menées par ces historiens, à parti d'études d'ouvrages, permettent ainsi de constater le caractère inépuisable des sources et, probablement, des investigations que l'on peut mener à partir d'elles. Cest ce qui mènerait ainsi à de nouvelles génération d'historiens qui actualiseraient perpétuellement de nouvelles mémoires.

Anthony Miche

\section{MEMORYLANDS. HERITAGE AND IDENTITY IN EUROPE TODAY}

Sharon MacDonald

London: Routledge, 2013, 293 p.

66 emory has become a major preoccupation - in Europe and beyond - in the twentieth century and into the twenty-first. [... Europe hasbeco tion" (1) it is with the iner (1). it is whese insightul words that cultura book Memorylands. The book is an opisiner investibook Memorylands. The book is an original investiand memory in contemporary Europe. In it MacDonit provides the reader with numerous examples of how provides the reader with numerous examples of how texopean identity and memory are constructed, contextraling all this in a vast and interdisciplinary and The fisthaf of the bophy.

The first half of the book concentrates on how the European past is nowadays made and narrated, felt and then sold. Having introduced the subject of her 
plex, MacDonald first illustrates how anthropologist iscussed the European past in their scholarly work dist identity and EU symbonism - from the EU anthen to to the a isplayed on Euro curse thropos in the study of Europe disisto antopolin warie tapics and topics as diferent as collective identity in the rura Argonne region of France or post-Yugoslav Bosnian are at the centre of the following chapter, where the are at the a ory, especially objects, food and homes. fromgardening as a way of re-rooting memory among Greek Cypriot per the mery porary Germany. As MacDonald notes, the memory phen to produc lo to of produced for tourists. This is the case of the heritage enve in 1993 or of the Slow Food move Scottish Is in Ita in 1996 and conlow For in Italy in 1996 and concerned with the pre

In the authenitic food products.

In the second half of the book, MacDonald anayzes specific dimensions of the memory phenomenon: musems a cosmopolitanism and lasty the future of memory Europen mering are a cor conpont of European memorial landscape and, according to the

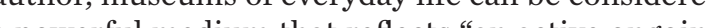
a powerful medium that reflects "an active ongoing the "the museatization of "ife" (141) and various anteur and " (141) and various anateur and local institutions such as the Latvian Elhnographic Open-Air Museum he the chaptens doul wist bring e to migration and the changes that migrants bring about in European identity, and then Holocaust memery into - accounta controversy enpted in Vienna in 2007 - when a statue representing a nude of a veiled woman was publicly displayed in the park of the Kunsthalle Nationale de l'Histie de l'Immigra Natin de lHistorede lingmigration of Paris, so to late new and more inclusive ideas of Europeanness. Holocaust commemorations are then interpreted as part of the larger morations are then interpreted as port citizens alike, that for European and non-European citizens allke, that might contribute to reconfigured ways of being national. The future of memory in understers ceris pers this whe the will no whe Cull no doubtemerge in Europe's nemorylands" (235) Cure co to confirm this suggestion, highlighting the need for ret

Even though Memorylands is largely based on articles originally published as separate papers, the autho managed to assemble everything in a well-readable nd coherent way. Inevitably in abook onsuch a broad subject, and as the author herself acknowledges in the . TacDonald conducted fieldwork - feature where macDonald conded nently, as opposed for example to Italy or Spain to would have liked to dedicated. In terms of topics, of the memorial lo rad a more detalled discussion with remontially memoration of the Algerian War, which ciena profound imper on idian War, which clearly have a profound impact on ideas of European identity and have have also allowed MacDonald to better clarify how the memory phenomenon is not entirely unique to Europe and West, as comparable scenarios can be found in locations su

is said, Memorylands is a timely and useful text that gives the readers a rich overview of the contemporary European memory phenomenon, while also in order to much of the scholarly literature needed in order to und osstantial reading for students and scholars interested in memwell as in anthropology and cultura history.

Dario Miccoli

\section{STORY OF A DEATH \\ FORETOLD: THE COUP \\ AGAINST SALVADOR ALLENDE, SEPTEMBER $11^{\mathrm{TH}}, 1973$}

Oscar Guardiola-Rivera

New York: Bloomsbury Press, 2013, 496 p.

$\mathrm{n}$ the last few decades many scholars have examine the rise of Chile's President Salvador Allende and the subsequent coup against the democratically elected socialist. In the context of the Cold War and revolution, Allende's notion of resistance or la vía chilena models. Within the fonfining conventional Cold War War, the War, the global conmunity viewed Latin Americans as having to choose between U.S. capitalism and Soviet communism. To many LatinAmericans neither of these alternatives provided truedin American undoubtedly offered Latin Americans a framework to critique U.S. imperialism, the left in Latin America consistently created their own dofo the find mocracy. Indeed, in 1970 Allende became a derst Marsist in history elected as the president of a democracy. Alde's and his way of, as GuardiolaRivera clains, "divorcing denocracy and law from Cold Wa bi , and pilvilege" moved beyond stifling the most bignificant exmples of homegrown one of the most significant examples of homegrown revoluworld view A of change inspired by particular his within Ce in within Chile and the experiences of Salvador Allende. the US. govercent his Pop gor Unity party as a daserous challenge to US Popular Unity party as a dangerous challenge to U.S. hegenon and capitalism. In hiswell-researched monograph, Guardiola-Rivera provides one of the to pow the to power, the coup again $\mathrm{Ch}$ ile this tragic event within Chile.

Guardiola-Rivera's work tells the story of Allend in three parts. The first concerns precedents an causes of Allende's rise to power. Guardiola-River how this in inired him to tive for the people" (12). This inspiration came from both theoretical influences and real life interactions with political repression and revolutionary violence. differife experiences inspired Allende to envision influent influences reflect the hybrid and creative character of Latin American politics and revolution. In this section Guardiola-Rivera alsolinks Allende's ideological influences with the greater political climate within Chile This rienced within his lifetime and also another layer to what could have been astandard biography. The book particularly strong when Guardiola-Rivera references

In part II, the Coup, the auth che Guar

In part 11, the Coup, the author focuses on the vevents leading up to Allende's election and the 1970 Rodrí In the bez a rog In the begining of this section Guardiola-River Unity party in the lor che Unity party in the laryer context of the Cold War. He Mapuche pop Alation As president Alles indigenous Me puche promised the people he would "change the regime and system

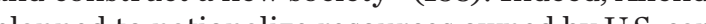
planned to nationalize resources owned by U.S. captop ung stop unemployment, and implement true agrarian War proveds promise within the context of the Cold War proved much too threatening to U.S. hegemony. Chilean military officers with the hope of oroch ing a coup. Guardiola - Rivera uses CIA doctestrating a coup. Guardiola-Rivera uses CIA documents US. was in subverting Allende's demly involved the In this sion, which in In this section, which in some ways parallels Greg ra in the Cold War (University ica in the Cold War (University or Chicago Press, 2004) Guardiola-Rivera explores ideologies about human Guard that ener out of global post-war politics. Guardiola-Rivera argues that so-called develope rights but only within the narrow context of "civilized rights but only winin the narrow context of "civilized nations". According to both Grandin and Guardiola-

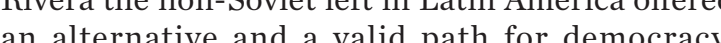
an alternative and a valid path for democracy. Therefore, in spite of claiming to support democracy, throw Allende even after a second election reinfored his legitimacy. Th 
against Allende was a coup against "law and democracy" (238). Indeed, human rights did not matter whe che so-called cillized ally powerful whe world section description of the eventsleading up to the coup and the

Sections suent take over.

Sectionsequences, which is shorter than the other sections, analyzes politica action in Chile up to the present. It examines the rulin Junta and General Augusto Pinochet's "destruction (340). Gular movement, and popular legal politics" "(340). Guardiola-Rivera explores the two types of "and the subsur and the subsequent destruction or human rights. The to ever eve "su Latin Anerica dung the Cold War. He - whether soldiers, civil ent lets no one off the hook means the individual liability of human rights viola(35). This powerful statement, hike others in Guardiola-River, work, connects thecoup against Allende to larger his$20^{\text {th }}$ century.

Though the book at times turns into a hagiography of Allende, Guardiola-Rivera's passion for his subject evident. Thisbook wants to go beyon ageneral Allende bibliography and influences within the complex history of Chile.

Lindsey Churchil

\section{WAITING FOR MARCEL \\ ORPHÜLS: THE LITERATURE \\ OF AUTHORITARIAN RULE AND RESISTANCE IN LATIN AMERICA}

Becoming the Tupamaros: Solidarity and Transnation Revolutionaries in Uruguay and the United States Revolutionaries in Uruguay and the United Sta

Nashville: Vanderbilt University Press, 2014, 206 p.

The Ideological Origins of the Dirty War: Fascism Populism, and Dictatorship in Twentieth Century Argentina

(n)

Oxford: Oxford University Press, 2014, 216 p.

Memory and Transitional Justice in Argentina and Uruguay: Against Impunity

New York: Palgrave Macmillan, 2013, 319 p.

2010, at the XXIX Latin American Studies Association Congress in Toronto, the historian Steve Stern called 1970s stated-sponsored terror in Argentina a "dirty war". An audience member self-identified as an "Argentine", speaking for "Argentines" scolded him. "Dirty war", he told Stern, was a term invented by the Argentine dictatorship, and as such, should never be used. Stern, perhaps the most authoritative analyst of Augusto Pinochet's Chile, apologized, conceding a truth on that policing language brings us closer to atruth on state terror. The moment evoked the mannertise those who say "Holocaust" for their putative chastise those who say "Holocaust for their putative the Nazis.

As the international scholarship on authoritarian violence in Latin America has grown quickly since 1990, dominant narratives have tended often to rigid binaries on violence, morality, state terror, and even language. As in the use or the term "dirty war", there i investigators racing to fit their cases into these dis- courses - like round pegs into square holes - have disregarded what to an outsider may seem obvious based on the evidence. Commenting on a museum opene in 2007 to remember victims of the 1968 Tlatelolco Massacre in Mexico City, for example, the literary critic José Ramón Ruisánchez Serra writes, "despite a genuine effort to create a site where the most cruevent in the second half of the twentieth centu in Mexico could be commemorated [...] the museun receives few visitors". (Ruisánchez Serra, José Ramó Reading '68: The Tlatelolco Memorial and GentrifPayne (eds) Accounting for Violence: Marketing rayne (eds.), Accouny (2011, 181) In trying to expla visit this ln trying to explain why Mexicans will not to Ruiśn nez Serra that his starting it never occurs Run hould it be that his starting prenise nay wong. Could it be that the power ucadenic line that Tlatelolco was both a un state ter mold War Mexicois inacurate? is it possthe because they do not view Tlatelo

Much of the academic communty addressing Much of the acadenic community addressing authoritarian violence in 1970s and 1980s Latin America has nothad a MarcelOrphuls moment. Some can't see grey zones on authoritarian inle - that the tern "dirty war" can have multiple meanings or the che ent Mexicans. This is reminiscent of French politipitié (1969) prompted an opening in how the French pitie (1969) prompted an opening in how the French 1960s, a do 1960s, a doming te Gaullist nave admitted only onemenory those who supported the occupation and there were those whory likely helped Socisist The persistence of that binary likely helped Socialist president François dectes: during the Second World War he had been both a ming dis both a menber of the resistance a in a Vichy bureaucrat, a combination so seemingly incongruous and so at odds with post-war French memory making on the 1964, that wo 1964. As French analysts have come to understand, not only can the Mitterand conundrum not be swept under the carpet. It is precisely that appant contradiction faring
Of the three books reviewed here, two take us long way in breaking down false binaries an dictatorship-era shibboleths (the third less so). Thei mportance rests in the first instance in a rejection of the powerful tendency in the scholarly literature to treat dictatorship as aberrant. They explain violen military rule (and resistance) not as some Frankentein's monster emerging from American Cold Wa nti-Coment 作 long term Lindsey Churchill's Becoming the Tup the ros the Tupamaries between military rule and its opponts (a) case, the leftist guerrillagroup, Tupamaros). This is no horoico for authoritarian violence or a maligning of and empirically with the hypothesis that to take as a and en the Cold War oppostes is and

Becoming the Tupamaros delves into the often poorly explained gendered abuse and humiliatio of political prisoners in dictatorship-era Uruguay. Che pexualized viothe against women Tupamaros. She also documents torture methods. But the book also explains both the violent military and the Tupamaro resist both the violent military and the Tupamaro resistance as in Uruguan society. At the same time, Churchill in produs th of long prociety. In their clandestine publications, for ple, the Tupamaros excoriated military authorities for pe, the Tupanaros excoriated nilltary authorities for mprisoning maleguerillas in ageneral prison population that included gay men. The result, according to the f red from a broken window. The Tup a maro was punished with yers of sitory. The Tupanas mental illness. In this and other cases, the Uruguay

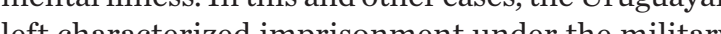
eft characterized imprisonment under the military and "predatory" "ay men. and "predatory" gay men.

Both inside and outside prison, the revolutionary left in Uruguay and elsewhere in Latin America fre quently shared a prejudiced view of homosexuality weyond this, Churchill wites, "like the Uruguasn 
military [...] the Tupamaros supported the polarizaion of masculine/feminine and active/passive, wit eminine and passive as synonymous" (Churchill 2014, (5). Thesons of so-called sexual deviance across political divide reflected longer term continuities. Like nonwhitenes homosexuality seemed invisible in post-dictatorship com violence.

Churchill also points out other continuities and connections between post-dictatorship democracy, the thing overingping political cultures is as importhe Tupamaros as progressive on the integration of women into political activity, upending notions of women as ate time, the revolutionary left in Uruguay asked same time, the revolutionary left in Uruguay asked won ton th, and to becons socially constructed nodels of nilitant masculinity. Other leftistgroups stressed women's remininity an aternity or embracing "enbracing eninity and maternity or embracing masculinity dichotomous gender restrictions confined women's political participation to const

With the fall of the

With the fall of the dictatorship in Uruguay an ven more so in Argentina, an initial reconciliation ence far right and the far left. Churchill raises another key longthe rontinuity through dictatorship by explaining pere resto eaceulden ne of their nubly for 2009. Remarkably for some, on the campaign, Mujica . 156). So 156). Some authors have tied the Tupamaros transton to electoral politics in the context of similar developments in Nicaragua and El Salvador. Churchill has the dicespip the Tupaman were always as much "Uruguaya" the Tupamaros were always as nuch "Uruguayn" as they were Cuba-inspired revolutionary Marxists. The nomic elites and church ties to politics produced wh

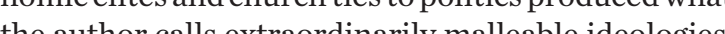
This proved true not only of democratic politics, but of

the Tupamaros' unique ability to mix conflicting ideologies into praxis. In the early 1970s, many members of the Tupamaros had no qualms "expressing admiration 159). Uhe (Churchill 2014, 159). Unlike other leftist revolutionary groups of the era, the Tupamaros never rejected everything abouthe backdrop to the widespread popularity across ideologal boundaries of the Mujica presidency.

Also dismissive of the simplistic notion of dictatorship as a Cold War deviation, Federico Finchelstein's Ideological Origins offers a compelling and bold new answer to the question, was the military government fascist? In post dictatorship Argentine popular culUnited States, there was a strong tentorship in the the regime with Nazism or fascism. to the dic with Nazism or fascis. That approach to the dictatorship drew, first, on a tendency among some to isolate the period as a a te interruption to modern, civil rule that must neve be pernitted again. In adition, it was based on the characterization of the regine as Nazi/fascist by some in the international han ticularly in regard to the detention and torture of the Jewish jour in own famous characterization of his ordeal as a descent nto a new Nazism.

Finchelstein's linkage of dictatorship to fascism is more thoughtful and compelling. An aberrant, violent historical moment did not define the dictatorship as fascist, nor did the mistreatment of Timerman and other Jews in and of itself. The regime's fascist tendencies have their origins in longstanding Argentine ideological and political traditions extending back at least through the $1930 \mathrm{~s}$, and transcending both democratic and dictatorial periods. This is especially evident in the construction of an internal, subversive enemy that concealed a foreign, intrusive presence. Drawing on 1930s right-wing tropes, at the time of the 1978 FIFA World Cup of Soccer (held in Argentina), "the widy a "the widely accepted fantasy that the enemy was not (Finchelstin or only Argentine by circumstance (Finchelstein 2014, 150). Reinforcing the notion that soccer was linked to the dictatorship and longstanding fascistic ideals of Argentine nationalism, the coach of junta leader and psychopuis Menotti thanked military junta leader and psychopath Admiral Emilio Massera the World Cup to Argentines as a defence of the rea Argentina against its enemies, where the dictatorship identified itself with the sacred and the hosting of the event as a victory over subversion.

Ideological Origins goes on to show another imporlink between military dictato

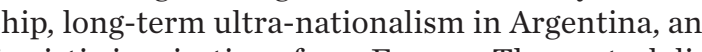
ascistic inspirations from Europe. The central dictatorship objective of defending national sovereignty from Nazi Ge non al war drew from Nazi Germany and fascist Italy. All at once, in cin winnable, and could never really be over. This new identify enemy was a the subse War becam a value. The fercity a foreign threat. Writheca War both molilized an enthe to the 1982 Malvinas drew dir mobilized an enthusiastic population and drew directly on years of ultra-right wing arguments the sak tha latter had long held that dying

Where Ideological Origins and Becoming

Where Ideological Origins and Becoming The Tupamaros dispense with dull binaries in trying to explain dictatorship not as aberrant but for its conJustice in Argentina and Uruguy is preorition Justice in Argentina and Unyuay is preoccupied with exactly the opp

The book asks, what is the relationship between transitional justice and memory? Francesca Less offers an unprecedented overview of theoretical an empirical literatures on amnesties, trials, truth commissions, reparations, and memory narratives. Her Stern [.] define te 'emble Stern [...] defined as "emblematic memory", a framewhtiple humes to give human efforts, conflictual and competitive, to give meaning" to collective, decisive, or transforpublicor mination publicor semipublic domains like the mass media, government events, street denonstrations and protests, music, books, and television [...]. [ [They are] socially constructed and selective, they are neither arbitrary inventions nor manipulations, but offer influential an convincing enunciations of often intensely disputed events (Lessa 2013, 20-21). Enblenatic or collective memory in Argentin

In a chapter on transitional justice in Argentin (still ongoing after more than three decades), Lessa is particularly taken with what she describes as the "political moment" represented in Néstor Kirchner's lection to the presidency in 2003. This marked a shift away from the impunity of the 1990s and into a new per new phase of judicial reckoning, there were 262 convitions on dictatorship-era crimes and counting. The judicial fy the the Río de a rata estuary to the hundreds of memorial plaques recalling sites from which people were "dis ther cities,

Chn Chapters on Uruguay trace asinilar uplifting story crls collo calls collective, enblematic memories were pitte gainst dictatorship impunity. Like Argentina, with he past decade a finaling coust in the late 1980s and 1990s "a memory of silen a blivion, with the state being totally silent on de and over the weed for truth, justice, repatriations, mem theed for truth, justee, repatriations, and As much as anything Memory and Transitional As much as anything, Memory and Transitional Jusfice is a morality tale of two civil societies that have benton the ling bent onjuxtaposing dictatorship and democracy, Less and memory then runs with them for the length and memory, then runs with them for the length of Carlos Menem for example, to the memory of silence and oblivion, like other critics of that ady of silence (once sensibly described by a feral judge as a illic (once sensibly described by a federal judge as an illicit che organization), Lessa assumes that the politics of men's two-term presidency was not a reflection in that the is till no st. Is that a credible assertion in that there is still no study of participatory politics period of den, whose presin that the panned a period of democracy as open as that of the past fifteen years? Is it possible that what Lessa characterizes as silence and oblivion was, in fact, a reflection of an alternative memory, sense of justice, and criminal wron uring and after military rule?

Beginning with her arguments on emblematic memories, Lessa routinely assumes that there is a
collective memory on dictatorship in Argentina and 
Uruguay that has finally triumphed as a result of the current wave of prosecutions. There is simply no evicer to rection of political cultures in wite, middle-class urban enclaves in Argentina and of the Kirchner prest-

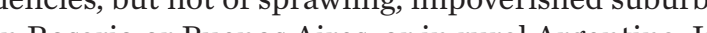
in Ras collective "Arsentine" memoris that a on cen "Ar fective for the white, urbaniddle class. Lessacites the 1090s impity instead of blaming the miltary and rov impunity, instead of blaming the military for thecond that narrative was overcome after 2003 (and blame squar "f laid at the feet of cher juctialaccountingand

Let's leave aside whether or not working Argentine living in the one million strong, largely impoverishe Florencio Varela municipality in Greater Buenos Aires ascribe or ascibed any blome for 1970 s politpore repres the poignant issue is the either-or binary of two demons

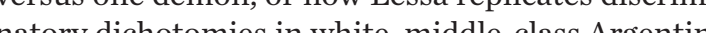
natory dichotomies in white, midde-class Argentine cories Nobody has everconducted astudyin wich work working people of colour in Varela and hundreds of Whercom "nstead, a dominant narrative that accompanies that or appropriate remembrance (that is to say, Lessa and Uruguayans a and Uruguayans across class, race, and geographical , don'thave theconect

While racialized class differences shape society hroughout Latin America, unlike Guatemala and Chile state terror in Argentina and Uruguay was not directed in the first instance at working people of color. In this not in symptathy with authoritarian rule, or especilly preocupied with whether violence came from the dicpreoceupied with whether violence came from the dictatorship or the guerrillas. Most remained distant from ith all regards and in a context where state violence was during the dictatorship, and in democracy afterward and without significant difference. In 2007, the world champion boxer Juan Martín Coggi remembered his riday evenings as a young teen-ager fighting in the 列 列 nuch bigger and stronger than him. He made money his improm is inprobatevicting. me, people asked why. He noted hat everybodyknew that Falcon whe what In intervain.

two hours and had covered the dictatorship period, this was the first and lives rence to military dictatorship touching the and millions of poor, working Arsentines of coggi, and niterror was incidental to their lives bearing state teror was incidental to therr lives bearing in mind ong term continuities of poverty and police violence. Their memories and narratives of dictatorship what thely unk widd midde-class nemory-making institutions (often lionized by academics) that their memory "oblivion" is ignor ance. For those who have yet to feel the impact of post-1983 transitional justice in their everyday lives, thro-human rights Are discin. The architect the new, pro-human rights Argentina, president Raúl Alfonsin, died in 2009 . When newspapers reported on the work of his post-dictatorship human rights heroics, one Buenos Aires resident who had lived through intense pove funced at the She was evidenty stuck in oblivion She was evidently stuck in oblivion.

David M. K. Sheinin

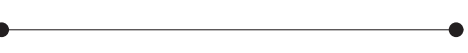

TO WAR. A JOURNEY ALONG THE FRONT OF WORLD WAR I

Arnout Hauben \& Johanna Spaey

Antwerpen: De Bezige Bij, 2013, 272 p.

nfotainment is supposed to disseminate historic knowledge to a broader audience, and I am fully aware that this unavoidably implies the loss of some nuance in favour of the genre's entertaining aspect. Th problem with Arnout Hauben and Johanna Spaey's

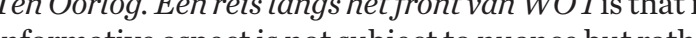

A few preliminary remarks. Reading the title raise some suspicions: the subtitle, A Journey Along the Fentline of World War 1 , niplies that the focus will be solely on the Western Front in Europe, neglecting the Easter Front, the clashes in Africa and Asia, and iven, at sea. It also defines the fronthe as a static given, whichis an exageration. Yes, the Western Front f 1914 and the year 1918, with both the Kaisersch and the year 1918, with both the Kaiserschlach

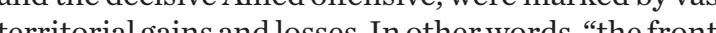
tern" is a bins and losses. In other words, "the fronten or a even, or especially, when ane Aside from that, hisingsenthen tate to call the television programme a documentary a con that the

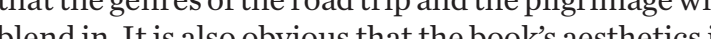
hily. In heavily infuenced, via the TV show, by the visual arts: the im a horiztate as the image of a horizon to depict a clear and complete overview. The question is, hhen, how these genres and aesthetics the theoks docume far claims. Luckily the authors are aware of their at once extensive and limited approach. They do signal that the Western Front was one of many war theatres (18) and that (Belgian) know of front and the campaigns in the Balkan fo the Italia front and the campaigns in the Balkans form an innovative inclusion for a Belgian target audience. The ans and of was not only fought by young white men. Hence, it does

also makes explicit claims of causality as to the infuence mort as Canad (56) and Italy (138), in the line of John as Canada (56) and Italy (138), in the line of John the Somme on post-war British society in The Face f Battle (Lon of Battle (London: Pimlico, 2004). As such, it touches con the relevance of the First World War and its con

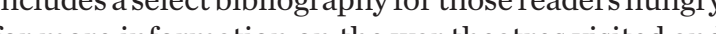
the individuals' lic stois mentioned.

Finally, the book is unsurprisingly reader-friendly and at times attempts to include an authentic and at thes attenpts to include an authentic documents, such as letters home and diary entries, documents, such as letters home and diary entries, to undermine the contemporary war popaganda by iving a voice to its victims, wishing to illustrate conflict's conting their tis the description of an elderly couple, wholost collecting WorldWar I hells a a kind of cly the shells may be seen as a "transenir egy. The shells may be seen as a "transgenerational doomed to fail. "Deberg mot is coon to fail. "De bergmoet immens zijn, net zo groo as huse f what the authors attempt on may try to conp of what the authors attempt: one may try to come to created but ultimately the dead remain this past has

Yet this emotionally powerful insight is rendered a dentifict identification with the soldiers on the frontlines. Empathy is a necessary attitude when undertaking draws between his but the paralls that Haube mise ensène of their departure is telling a fanfare ise playing the thas post while the crew of thare band is playing the Last Post, while the crew of three kisse "zols "zoals elkejonge soldaat die in de Eerste Wereldoorlog "zoung soldier (

Another scene, in which Hauben draws a paralle between the artillery fire directed at Reims in 1914 metar ther metapharcalcons the the 
expectations regarding the participatory nature of ats ance and attention to the historical dimension is required.

However, this problematic attitude is in my opinion not the book's greatest flaw. Even more aggravating The the the byptual errors and contradictions. These are the byproduct of the attenpt to inustrate te feads to the usage of contradictory naps or picture fom ancther context, which smacks of an uncritica hor pex Also, the depiction of the frontines east of Rims is Also, the depiction of the fronthines east of Reins is inexact: the map (78) indicates the trenches ran from

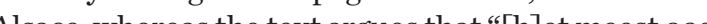
telijke deel van de Franse frontlinie wordt gevor

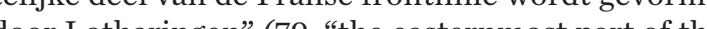
con rench frontline is shaped by Lorraine"). One may 1871 until 1918, yet this is not indicated on they from s7intilis ntioned in the text.

Another example of this impreciseness is the dicrion ofthe Belgian sector in the frontline around Diksmuide, "in Somme, two years later. (32) Fingly, out le or somme, two years later. (32) Finally, on at least one Cermen touristsearwitness to the destruction of Germantoursts ba II to the destruction of Germany during World War II, supposedly by American Spitfires were British fighters, nat American bo po the Ger Bitish fighters, not Anerican on to the German survivors this makes no difference of course, but ou the authors. Evidently it would not be very tactful to confront the survivors with such errors in the minieflect on the ex how doct on the extensive use of eyewitness accounts in do Add some min mistakes, such

Add some minor mistakes, such as a translation error in Rudyar Kipling's My Boy Jack (71) and the provion of presumably lesser-known facts without providing background information (e.g., the presence his boup live up to its this book canctive up to its documentary claims. in documentary fiction, such as the oeuvres of W.G.

Sebald and Alexander Kluge - they even constitute the backbone of the authors' poetics. The problem with Spey and Hauben's narrative is, howe problen with Spaey and Hains aris the contradictions fiction but as a reportage. Hence, appeal to the reader's persicacity. The bor is rathe appeal to the reader's perspicacity. The book is rather minis light-hearted the this this respect, as is the pilgrimage. the crew of three pays homage to those fallen and those marred during and after the war and thereby continues the tradition of ough alys of the wars cas sacrosanets. A thorough analysis of the war's causes and consequences is nearly completely absent, and the unprecedented brody nature of the war is blamed on the high-tech art of warfare - not mentioning the treatment of POW In spite of the inclusion of overseas topation. In spite of the inclusion of overseas troops and labourers, the (very few) Congolese troops on Belgian soil are - rather oddly - not mentioned, nor are the Moroccan or Algerian forces in the French armies, whose descendants (also) live in Belgium. Therefore, his book misses out on the unique opportunity of telling the war stories of Belgium's new citizens' ancestors Again, they are denied access to our (Belgian) practices ing to the all-soldier-are-sing them and by adherfail to reflect on their own which is an the which is a shame. The interest of a broader audience would the inve waned, since the books road-trip structhe various emotionally powown war theatres, and have offered the perfect counteru anechotes would have une remarks. Dry, because on a meta-lry and uneasy remarks. Dry, because on a meta-level; view on World War I, and may hint spots within our wite

In general, this book had a lot of potential, but suffered from the authors' inaccuracy and uncritical position, which undermines the credibility of their work Thist but it also illustrates how easily such events conbecol become persistent myths. Surely, a methodologically have roulted in a True and Final Story. Bus would no hould huled in a True and Final Story. But at least it events. Instead, the do inting ention of historical is, sadly frustration

\section{THE SLEEPWALKERS \\ HOW EUROPE WENT TO WAR IN 1914}

Christopher Clark

London: Allen Lane, 2012, 736 p.

014 is not just a year like any other: it carrie the commemorative weight of crisis, war, and eration, freedom and new beginning 2014 repsents the jemus $\mathrm{faced}$ character of the modern era here life-changing innovations evoked alongside whe life-changing innovations evoked alongside f three significat dates in of three significant dates in modern history brings hrough which abhorrentlossons wo whe live in today wh in to bein today came into being. This year, we commemorate ing of the American troops on the cost of Norm in of the Antican trops on the coast of Nornandy ine Wall 1989. All these dates matk plic ing public memory of contemporary Europe, and strongly iangle of 1914, 1944 and 1989, Europe was born as a triangle or 1914, 1944 and 1989, Europe was born as a reaction to the to to the bink destruction twice found oundations were laid to (soen. In 2012 hist we know today.

In 2012, historian Christopher Clark published a monumental work on the origins of World War Europe Went to War in 1914 ale Sleepwalkers - How Europe Went to War in 1914 already suggests that Clark th thin tant spot within paradigmatic effort to name a culprit, to acuminate the ful and unmistak built Clark explicitly re dreadful and unmistakable guilt. Clark explicitly refuses to arrange the profusion of sources in a way which supports any teleological narrative. He writes: There the his work aims to retrace the steps which led to cerhis work aims to retrace the steps which led to cerquestions how the war broke out, not pin an questions how the war broke out, not primarily why. one decision affected the other. The result of this chain f reactions was - in its total scope and minacious out come - unrecognizable to the key players at the time. The Sleepwalkers closely examines the structures of power within Europe, poses questions of where thi actual power was executed and where not, and thu challenges the notion of "steadily building causal pressure" (xxvii) which led to an "inevitable" war

In his introduction, Christopher Clark starts with the time-and-again striking statement that it only took (harajevo assasi Archduke Franz Ferdinand and his wife Sophie, Duchess of Hohenburg, to the unleashing of an inferno that would extinct entire generations. Introducing his study with this statement might already raise awarenes wive reader that this rather awort time perod between the event and the outbreak could just be an excerpt, a detail from antterly more complex Clark describes what the historian encounters while claring to take un the his ying to take up the manifold argumentative threads hem into a cloth that lacks apologetic con a curces, which oftes. Faced with an oversupply volid infor which often present an undersupply of valid information, Clark describes a "world war of

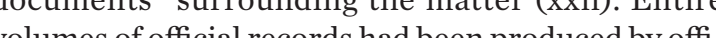
che the war was ended and the treaty of Versalles signed. The war was ended and the treaty of Versailles signed. these offial "ecords, however, are frequently chara derized by "endentious omissions" (xxii). Memoir cloly oction" proved valuable the lections" proved valuable, the protagonists were often concerned with self-justification (xxii). It is these key the epenemeral, but rather obscure power structure their respective countries that Clark carefully revisits.

The Sleepwalkers is divided into thrully revists. The Sleepwalkers is divided into three parts. The oping argument will undertake in the following. Part One readjunts ou "common knowledge" of the Part jevo assassination as being the point-of-origin of the first madern war.

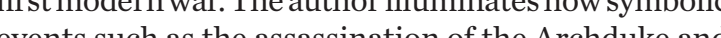
his wife were turned into stories, which toune an his wife were turned into stories, which took up a life nid own and witt exploited as nartatives on which before the was built. In fact, thesecd wasplanted long 
inherent political instability within the Balkan sphere which had been a reason for international confrontations for decades. Furthernore, he sheds a light on the politicaltensionswich anse states. In this first part, Clark nakesit abundantly clear that the orighs of war are located within continuing redentist discoures a lock the ticular. The latter was in orm dentity being grinded down within the multinationa commonwealthor Austria-Hungary. As Clarkremarks,

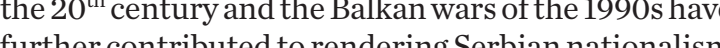

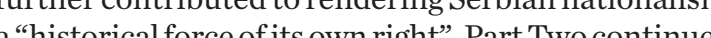
"histere the development of Clark's argument by a dissecting f maps, alliances, and international relations. The and alliances, and shows how they shifted throughout the des, and show how they shinted throughout the development of the crisis. Indeed, the GermanAustrian Treaty, the Franco-Russian Alliance of 1894 the Entente Cordiale between France and Britain in

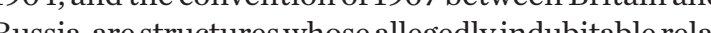
ussa, are structures inoseally tons are only visible in retrospect. Part Three count down the days and recounts the last decisions (or deciof a war which was framed to be highly imp

.

As Thomas Laqueur ('Some Damn Fooling Thing', London the title The Sleepwalkers does not support the poin that Clark is making her. In his introduction, Clark explicitly mentions the "watchful calculated steps" the months and "calculated steps" do neither constitute sleep). These "calculated steps" doneither constitute sleepwalking as far as my understanding of the concept goes, nor did Clark make any attempts to exonerate the protagonist Christopher Clark is a mions of Christopher Clark is a master of his sources, balance with representing scenes and episodes in ancerned with representing scenes and episodes in an almost to torte manner. The narrative is constructed not to topple over a language that keeps the reader at scientific distance. The author balances the proporthus dwe thus deploying methodology - and the steady flow of his beautiful prose. Given that Clark's point is that he walkers, his narrative is saturated with agency of the historian. However, Clark touches upon the role of the historian only in passing. He acknowledges that access to First World War narratives and the conclusions that we contemporaries draw are a matter of viewpoint, social and scholarly contexts, and experiences we (re) make. A discussion of his role, that of the historian, as an architect of history in the making seems utterly the the master narrative of exclusive German guilt. That is in itself a very postmodern endeavor, though something which Clark is unaware of or does not conside guilt is closty connected to Geran. The question of guilt is closely connected to Gernan self-awarenes indity identity were constructed as a reaction to this question. Clark this debate had on the reevaluation of Nazism and the Third Reich. Acommentary from the author on the disruptive, yet constructive potential of his work,

In his introduction, Clark already makes one of the most interesting points. He reframes the First World War as a "modern event". A modern crisis, in his terms, is characterized through the existence of "suicide thes shook the world during the 2000s. He goes on:

Behind the outrage of Sarajevo was an avowedly terroris organization with a cult of sacrifice, death and revenge, but this organization was extra-territorial, without a clear geographical or political location, it was scattered in cells across political borders, it was unaccountable, it. Inks to any sovereign government were oblique, hidden and certainly very difficult to discern from outside the organization. Indeed, one could even say that July 1914 the 1980 s. (xxv) in the 1980s. (xxv)

Yes, modern warfare, if you may, is characterized through scattered groups which base their raison-d'être on rather conservative world views and rigid morals, pretending to defend their formative principles. Clark's nodern enemy has become abstract, impersonal, a sleeper or fanatic amidst us. Clark did find some and the suicide warriors of the $21^{\text {st }}$ century. first and forme warriors of the 21 century. However, mod and forenost, in my opinion, the July Crisis is a the national concept. It heralded the end of the nation, for it carried the seed of supranational structures. A Clark has convincingly shown, the key decision makcond forged to prevent intrusions into the respecive hand, forged to prevent intrisions into the respective allional sphere, and alliances on the other. As a consequence, the entire doxically posed a loming the th its Itwo phadoxically, posed aloon acterized through mutual distrust and an anbiguity Tha tin ite That in itself might notbe a surprise or an extraordinary conclusion, but it already opens up a range of in particular with regard to contempory ofwar in particular with regard to contemporary controis a result of many of the aspects that Clion. Europe it is a un man o it is a supranational structire which seeks to bridge nationalinterests an provi in the years pringeding narrative that was absent in the years preceding the turou founding narrative has becof that European founding nan tive has becone more and Putin's opportunism hasled the European realmat putin's opportunim "improbab" brink or another "improbable" crisis. The Russiangovthe Ukraine and the EU might le the Ukraine and the EU might lead to a furthering of power ind to a strengthening of perceived Eunculture, and to a strengthening of perceived Europea cibly any. As aconsequenc, Putininterven and forcibly annexed the Crimea. The EU is clearly struggling

The rent success of anti-European poplis. The recent success of anti-European populis insts another dark clond over the pu project ing at an unsettling tendency to reiterate the concept of nationalism. Whilst one pean union, one aspect remains striking: although we might find ourselves in the era of many "posts" - post, posterinisn - the the nation is by no means deac. acing the altar of he European community became a mighty che in thes being ready to restrain the influence the EU on hational sovereignty disturbingly reminds us of how calla the weeks precenting whe Clark claims that supraplicy maingwere non-existing in 1914, and that the policy making were non-existing in 1914, and that the int Eres the european crisis we encounter today, many of those hips between the European players are again osillatships between the European players are again oscillatinteres interests. Although the public opinion seems to remain against European intervention in the Ukrainian crisis, the conclusions that Christopher Clark has re the conclusions that Christopher Clark has reache alarmingly remind us of the reasons why Europe exist doing so

Of course, the publication of The Sleepwalker predates the Ukrainian crisis. Nonetheless, one might Whant to pose these question to Christopher Clark: What are the implications of his work for the postWhat is the role of the historian in constrenting chat is the role of the historian in constructing and contesting master narratives and collective identities? which were a direct result of the World Wars? I 